\title{
The effectiveness of massage therapy for the treatment of nonspecific low back pain: a systematic review of systematic reviews
}

This article was published in the following Dove Press journal:

International Journal of General Medicine

3 September 2013

Number of times this article has been viewed

\author{
Saravana Kumar' \\ Kate Beaton' \\ Tricia Hughes ${ }^{2}$ \\ 'International Centre for Allied \\ Health Evidence, School of Health \\ Sciences, University of South \\ Australia, Adelaide, South Australia, \\ Australia; ${ }^{2}$ Australian Association \\ of Massage Therapists, Adelaide, \\ South Australia, Australia
}

Introduction: The last decade has seen a growth in the utilization of complementary and alternative medicine therapies, and one of the most popular and sought-after complementary and alternative medicine therapies for nonspecific low back pain is massage. Massage may often be perceived as a safe therapeutic modality without any significant risks or side effects. However, despite its popularity, there continues to be ongoing debate on the effectiveness of massage in treating nonspecific low back pain. With a rapidly evolving research evidence base and access to innovative means of synthesizing evidence, it is time to reinvestigate this issue.

Methods: A systematic, step-by-step approach, underpinned by best practice in reviewing the literature, was utilized as part of the methodology of this umbrella review. A systematic search was conducted in the following databases: Embase, MEDLINE, AMED, ICONDA, Academic Search Premier, Australia/New Zealand Reference Centre, CINAHL, HealthSource, SPORTDiscus, PubMed, The Cochrane Library, Scopus, Web of Knowledge/Web of Science, PsycINFO, and ProQuest Nursing and Allied Health Source, investigating systematic reviews and meta-analyses from January 2000 to December 2012, and restricted to English-language documents. Methodological quality of included reviews was undertaken using the Centre for Evidence Based Medicine critical appraisal tool.

Results: Nine systematic reviews were found. The methodological quality of the systematic reviews varied (from poor to excellent) although, overall, the primary research informing these systematic reviews was generally considered to be weak quality. The findings indicate that massage may be an effective treatment option when compared to placebo and some active treatment options (such as relaxation), especially in the short term. There is conflicting and contradictory findings for the effectiveness of massage therapy for the treatment of nonspecific low back pain when compared against other manual therapies (such as mobilization), standard medical care, and acupuncture.

Conclusion: There is an emerging body of evidence, albeit small, that supports the effectiveness of massage therapy for the treatment of non-specific low back pain in the short term. Due to common methodological flaws in the primary research, which informed the systematic reviews, recommendations arising from this evidence base should be interpreted with caution.

Keywords: massage therapy, systematic review, evidence-based practice, complementary and alternative medicine

\section{Introduction}

Recent times have witnessed dramatic changes to health care. There is now an overt recognition for quality to inform health care practices and this recognition for change has been driven by an increasingly well-informed consumer of health service, the patient, and other stakeholders who strive to underpin their service delivery within the 
quality health care framework. The key components of this framework include safety, effectiveness, patient centeredness, timeliness, efficiency, and equity. ${ }^{1}$ Much of the drive towards quality health care has been championed by evidence-based practice, which recognizes the need for health care practices to be underpinned by an integration of research evidence, clinical expertise, and patient values. ${ }^{2}$

Low back pain is one of the most common musculoskeletal disorders in modern society and is a major reason for health care utilization. ${ }^{3,4}$ The impact of low back pain is widespread, including physical, social, psychological, and economic aspects of an individual's life. Low back pain can include discomfort in any area of the spine from the 12 th rib to the inferior gluteal fold, and is only considered to be specific if its etiology is known (such as diagnoses of degenerative or other disease, infection, fracture, etc). ${ }^{3-5}$ Low back pain is usually reported as self-limiting (acute or subacute durations), but it is estimated that approximately $10 \%$ of this population will develop chronic pain. ${ }^{3,4,6}$ However, it has been suggested that this may be an underestimation, with the true number of low back pain sufferers who progress to chronic pain ranging from $16-62 \%$ at $6-12$ months post injury. ${ }^{7}$ This imposes a large burden on the health care system. ${ }^{4-8}$ In spite of a large body of research evidence and a plethora of interventions being available in this area, how best to manage this condition continues to pose a challenge.

The last decade has seen a growth in the utilization of complementary and alternative medicine (CAM) for a variety of health conditions, including musculoskeletal disorders such as nonspecific low back pain. ${ }^{3-9}$ Awareness in the general public about CAM therapies is growing and their use is becoming increasingly widespread. ${ }^{3,4}$ The total extrapolated cost in Australia of CAMs and CAM therapists in 2004 was AUD1. 8 billion. ${ }^{9}$ Common CAM therapies for nonspecific low back pain include acupuncture, massage, and manipulation. ${ }^{4}$

One of the most popular and sought-after CAM interventions for nonspecific low back pain is massage. ${ }^{4}$ There are many types of massage, including but not limited to Swedish massage; Thai massage (a form of body work involving assisted stretching); Shiatsu (a Japanese form of massage utilizing finger and palm pressure and stretching techniques); reflexology (the application of pressure to the zones of the feet, hands, or ears, which are thought to correspond to various body parts); and myofascial release (a manual therapy involving deep tissue work). Massage may often be perceived as a safe therapeutic modality without any significant risks or side effects $^{3}$ and has been recommended by the Chartered Society of Physiotherapy for the management of various pain-related conditions, especially those of musculoskeletal origin. ${ }^{10}$
Despite its popularity, there continues to be ongoing debate on the effectiveness of massage in treating nonspecific low back pain. While there is a large body of primary research evidence, such as randomized controlled trials (RCTs), historically, secondary research evidence such as systematic reviews often fail to draw any clear conclusions with which to inform health care practice and policies. With a rapidly evolving research evidence base, and access to innovative means of synthesizing evidence, it is time to reinvestigate this issue.

This systematic review of systematic reviews (umbrella review) sets out to provide a synthesis of the best available research evidence for the effectiveness of massage therapy for adults suffering from nonspecific low back pain. Systematic reviews are considered to be the highest level of evidence for intervention questions. ${ }^{11}$

\section{Methods \\ Review question}

This review determined the effectiveness of various forms of massage therapy on nonspecific low back pain in adults by answering the question "What is the evidence for the effectiveness of massage therapy in adults with nonspecific low back pain?"

\section{Types of participants}

Included participants were adults ( $\geq 18$ years) suffering from non-specific acute, sub-acute or chronic low back pain (low back pain is defined as pain that is localized from the 12th rib to the inferior gluteal fold)., "Non-specific" means that there is no specific cause of the low back pain such as neoplasms, infection, osteoporosis, arthritic conditions, fracture, radicular syndrome or inflammatory processes. ${ }^{3,12,16}$

\section{Types of exposure}

The treatment of interest in this review was massage therapy. For the purpose of this review, massage is defined as the manual manipulation of the soft tissues of the body for therapeutic purposes. , $^{3,5,12}$

\section{Types of comparators}

Comparators included but were not limited to: sham or placebo treatment, medical interventions, physical therapy, electrical therapy (transcutaneous electrical nerve stimulation, ultrasound, etc), pharmaceutical interventions, and other forms of alternative therapy.

\section{Types of outcomes}

Outcomes included but were not limited to: patient selfreport/subjective change of symptoms, assessment of 
pain, functional status as measured by validated tools, and assessment of range of motion.

\section{Search strategy Databases}

A systematic search was conducted in the following databases: Embase, MEDLINE, AMED, ICONDA, Academic Search Premier, Australia/New Zealand Reference Centre, CINAHL, HealthSource, SPORTDiscus, PubMed, The Cochrane Library, Scopus, Web of Knowledge/Web of Science, PsycINFO, and ProQuest Nursing and Allied Health Source, investigating systematic reviews and metaanalyses from January 2000 to December 2012, and restricted to English-language documents. The key words used to develop the search terms used can be found in Table 1. The appropriate truncation symbols and Boolean operators were used for each database searched and MeSH terms were used where applicable. Two researchers independently conducted the search with cross-checking of random databases to ensure consistency across the search.

\section{Pearling}

Reference lists of any umbrella reviews returned in this search were also pearled for additional systematic reviews or meta-analyses that may not have been found in the original search.

\section{Selection process}

Articles were included if they were systematic reviews or meta-analyses that addressed massage therapy for the treatment of nonspecific low back pain, as defined in this umbrella review. Massage could have been administered in either the treatment or control group, but information regarding massage groups had to be reported separately to any other complementary medicines or placebo treatments

Table I Concepts searched and the keywords related to these concepts

\begin{tabular}{|c|c|}
\hline Concept & Keywords \\
\hline $\begin{array}{l}\text { Massage } \\
\text { therapy }\end{array}$ & $\begin{array}{l}\text { Massage therapy, massage, remedial therapy, } \\
\text { acupressure, trigger point therapy, deep tissue } \\
\text { massage, sports massage, Swedish massage, therapeutic } \\
\text { massage, relaxation massage, muscle manipulation, } \\
\text { musculoskeletal manipulation, Rolfing, reflexology, } \\
\text { shiatsu, Thai massage, myofascial release, Bowen therapy }\end{array}$ \\
\hline $\begin{array}{l}\text { Low back } \\
\text { pain }\end{array}$ & $\begin{array}{l}\text { Back pain, backache, lumbago, sciatic, sciatica, spine, } \\
\text { spinal, vertebrae, vertebral, intervertebral, low back } \\
\text { pain, low back disorder, lower back pain, lumbar, } \\
\text { myofascial pain syndromes, scoliosis, kyphosis, lordosis, } \\
\text { intervertebral disc degeneration, disc prolapse, back } \\
\text { injury, back rehabilitation }\end{array}$ \\
\hline
\end{tabular}

administered. Articles that did not meet the PRISMA ${ }^{13}$ definition of a systematic review or meta-analysis were excluded, as were any previous versions of updated systematic reviews. Articles that investigated massage therapy as applied by chiropractors or physiotherapists were also excluded, as these practitioners are not classified as massage therapists and therefore do not fit the criteria for this umbrella review.

\section{Methodological quality assessment}

Once relevant publications were identified, two reviewers independently evaluated the methodological quality using the Centre for Evidence Based Medicine (CEBM) critical appraisal tool. ${ }^{14}$

\section{Data extraction}

The data were extracted into a custom-built table based on the CEBM questions. At this point, further exclusions were made based on the full text of the articles.

\section{Results}

\section{Search findings}

A total of 1,854 articles were returned in the initial search of the abovementioned databases, and two new systematic reviews were found by pearling the reference lists of other umbrella reviews. Of these 1,856 articles, 262 were duplicate articles and 1,393 were removed based on title, abstract, and study descriptors. The full text of the remaining 201 articles was assessed by two independent researchers for relevance, resulting in the exclusion of a further 192 articles that did not match the inclusion criteria. Any disputes were sent to a third researcher for arbitration. The remaining nine systematic reviews were included in this umbrella review. Figure 1 provides an overview of the selection process using the PRISMA flowchart format. ${ }^{13}$

\section{Methodological quality of included reviews}

The nine included systematic reviews were critically appraised with the CEBM critical appraisal tool. ${ }^{14}$ The overall quality scoring of the nine included systematic reviews ranged from poor to excellent. Table 2 provides an overview of critical appraisal scores for individual reviews.

The identified systematic reviews classified their findings into three categories of low back pain: acute, chronic, or mixed (where acute and chronic were reported together). In order to maintain standardization and keep true to the 


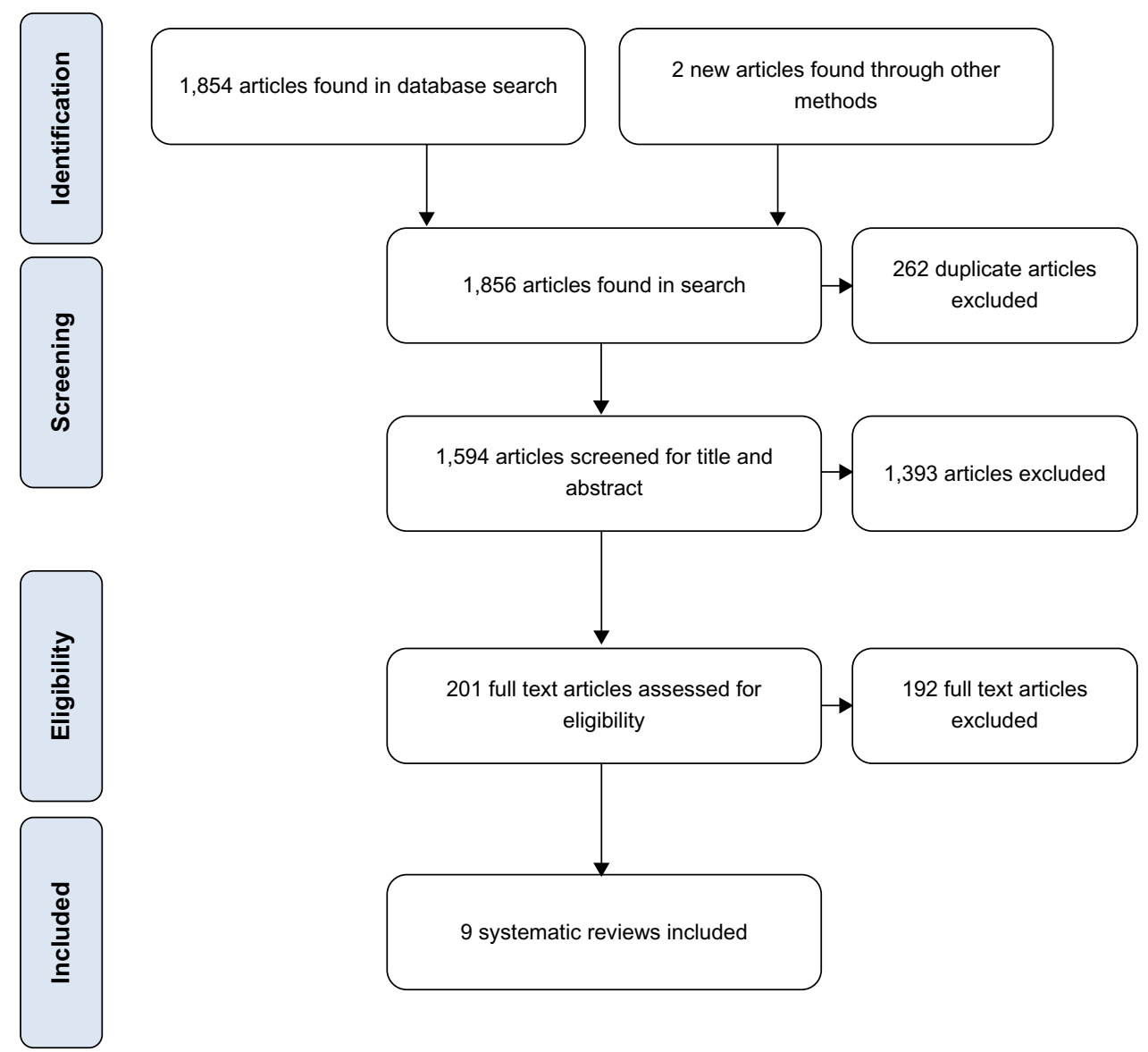

Figure I Flowchart of study selection.

Note: Flowchart is as per the PRISMA flowchart format. ${ }^{13}$

research evidence, the following section summarizes the findings based on these three categories.

\section{Category one: the effectiveness of massage} therapy for acute/subacute nonspecific low back pain in adults

Furlan et al ${ }^{4}$ identified ten trials, comprising 1,424 participants, that focused on the effectiveness of massage for low back pain. Of these, two trials were on acute/subacute nonspecific low back pain and included 158 participants. These trials showed significant short-term posttreatment benefits on pain and disability measures after massage when compared to placebo or no treatment. The assessment of methodological quality was only reported overall and the potential bias in massage-specific studies cannot be reported. However, it was reported that, overall (ie, in studies of acupuncture, massage, mobilization, and spinal manipulation), the quality of the studies was poor.

Table 2 Score of included articles as per the Centre for Evidence Based Medicine review validity appraisal sheet ${ }^{14}$

\begin{tabular}{|c|c|c|c|c|c|c|c|c|}
\hline Author & Year & QI & Q2 & Q3 & Q4 & Q5 & Q6 & Quality score \\
\hline Bronfort et al ${ }^{16}$ & 2004 & $\mathrm{Y}$ & $N$ & Y & $N$ & $N$ & Descriptively & Poor \\
\hline Brosseau et $\mathrm{a}^{20}$ & 2012 & $Y$ & $U$ & Y & $Y$ & $\mathrm{Y}$ & Descriptively & Good \\
\hline Ernst et $\mathrm{al}^{17}$ & 2011 & Y & $\mathrm{Y}$ & Y & Y & Y & Descriptively & Excellent \\
\hline Furlan et $\mathrm{al}^{4}$ & 2012 & $Y$ & $Y$ & $Y$ & U & $\mathrm{N}$ & Descriptively & Good \\
\hline Kim et al ${ }^{18}$ & 2012 & $Y$ & $\mathrm{U}$ & $Y$ & U & Y & Meta-analysis & Moderate \\
\hline Lewis and Johnson ${ }^{10}$ & 2006 & $\mathrm{Y}$ & $N$ & $\mathrm{~N}$ & $\mathrm{Y}$ & $N$ & Descriptively & Poor \\
\hline Lin et $\mathrm{a}^{23}$ & 2011 & $Y$ & $Y$ & Y & $Y$ & NA & Descriptively & Excellent \\
\hline Pengel et al $\left.\right|^{15}$ & 2002 & $Y$ & $\mathrm{U}$ & Y & $Y$ & $Y$ & Descriptively & Good \\
\hline van Middelkoop et al ${ }^{19}$ & 2011 & $Y$ & $\mathrm{U}$ & Y & $\mathrm{N}$ & $Y$ & Descriptively & Moderate \\
\hline
\end{tabular}

Abbreviations: $\mathrm{N}$, does not fulfill criteria; $\mathrm{NA}$, not applicable to the paper; $\mathrm{Q}$, question; $\mathrm{U}$, unclear if it fulfills criteria; $\mathrm{Y}$, fulfills criteria. 
Pengel et a ${ }^{15}$ identified two high quality RCTs, comprising 164 participants, which compared the use of massage to spinal manipulative therapy and corsets to treat non-specific low back pain. It was reported that spinal manipulative therapy and corsets both improved disability scores in comparison with massage (effect size 1.5, confidence interval [CI]: 0.8,2.2; effect size $-0.9 \mathrm{CI}-1.6,-0.1)$ when using the Roland Morris Disability Questionnaire (RMDQ).

Therefore, to summarize this research evidence, it is likely that massage therapy may offer some positive benefits in terms of reduction in pain and disability in the short term, when compared to placebo or no treatment. However, this was not the case when massage therapy was compared with spinal manipulative therapy and corsets.

\section{Category two: the effectiveness of massage therapy for chronic nonspecific low back pain in adults}

Bronfort et $\mathrm{al}^{16}$ identified one RCT, comprising 164 participants, which investigated spinal manipulation therapy and various forms of massage therapy for the treatment of chronic low back pain. The findings from this RCT, of low methodological quality (38\%), indicated that massage therapy was less effective than spinal manipulative therapy applied by a chiropractor for disability outcomes (statistically significant), but not for pain improvement (statistically nonsignificant).

Ernst et a ${ }^{17}$ identified two trials, comprising 249 participants, investigating reflexology for chronic low back pain. The authors assessed the methodological quality of the two trials they found using the Jadad scale and found that one of the RCTs was moderate (3/5) and the other was high quality $(5 / 5)$. Results from both included trials were similar with no significant change found between groups in either trial. While effects were found in one of the two included trials, this was not significant for reflexology in the treatment of low back pain. This systematic review failed to provide evidence that reflexology has benefits beyond that of a placebo effect. Ernst et al state: "In conclusion, the notion that reflexology is an effective treatment option is currently not based on the evidence from independently replicated, high-quality, clinical trials." 17

Furlan et $\mathrm{al}^{4}$ identified ten trials, comprising 1,424 participants, which focused on the efficacy of massage for low back pain. Of these ten trials, eight investigated chronic nonspecific low back pain in 1,266 participants. The evidence indicated that massage was either no different or better than mobilization in terms of immediate and intermediate pain intensity (Short Form-36 pain scale,
McGill Pain Questionnaire [two trials]) and disability (two trials), determined using the Oswestry Disability Index and RMDQ. According to two meta-analyses, massage was significantly better in terms of pain reduction (using the visual analog scale [VAS]) in comparison with relaxation and physical therapy (defined by Furlan et $\mathrm{al}^{4}$ as exercise and/or electrotherapy) immediately following treatment, for those with chronic nonspecific low back pain. In terms of the intermediate effect of massage for patients with chronic nonspecific low back pain, there were no significant differences in disability (according to RMDQ) or pain (VAS) when compared with usual care (exercise and advice). Furlan et al ${ }^{4}$ also found one trial indicating that massage significantly reduced pain intensity and disability in both immediate and long-term follow-ups compared to acupuncture. The assessment of quality was only reported overall; the potential bias in massage-specific studies cannot be reported. However, it was reported that, overall (ie, in studies of acupuncture, massage, mobilization, and spinal manipulation), the quality of the studies was poor.

Kim et $\mathrm{al}^{18}$ identified two RCTs, comprising 275 participants, which reported on the use of acupressure (pressing acupuncture points with a finger or device) for the treatment of chronic low back pain compared to routine physical therapy. Both trials showed significant effects on pain reduction compared to the routine physical therapy. A meta-analysis was conducted for the two RCTs reporting pain intensity posttreatment (4 weeks) and at 6-month follow-up for acupressure versus routine physical therapy for participants with chronic low back pain. This revealed that acupressure was more effective than physical therapy at 4 weeks. The authors state:

... meta-analysis demonstrated acupressure to be superior to physical therapy in terms of pain $[\mathrm{N}=275$; SMD -0.71 ; 95 per cent $\mathrm{CI}-0.96$ to $-0.47 ; P<0.00001$; heterogeneity: $\chi^{2}=0.15, P=0.70, I^{2}=0$ per cent $]$ after four weeks posttreatment. $^{18}$

At the 6-month follow-up, acupressure had a significant effect on pain, but the authors report presence of heterogeneity in the data analysis.

van Middelkoop et a $\mathrm{l}^{19}$ identified three low-quality RCTs, comprising 163 participants, which indicated that there was no statistically significant reduction in pain when the massage groups were compared with the control groups (relaxation therapy and acupuncture; pooled weighted mean difference was -0.93 [ $95 \% \mathrm{CI}-8.51]$ [sic]). They conclude there is insufficient good-quality data with which to come to a firm 
decision on the efficacy of massage therapy in the treatment of chronic low back pain.

Therefore, to summarize this research evidence, massage therapy may offer some positive benefits in terms of reduction in pain and disability in the short term, when compared to relaxation. However, this was not the case when massage therapy was compared with spinal manipulative therapy. There is equivocal evidence of effectiveness of massage therapy when compared to mobilization and usual care (advice and exercise).

\section{Category three: the effectiveness of massage therapy for mixed acute, subacute, and chronic nonspecific low back pain in adults}

Bronfort et al $^{16}$ identified three RCTs, comprising 197 participants, which investigated spinal manipulation therapy and various forms of massage therapy for the treatment of both acute and chronic low back pain. They found evidence to suggest that spinal manipulative therapy may be more effective in reducing pain (nonsignificant findings) than placebo massage, and a single session of spinal manipulative therapy resulted in fewer sick-leave days than friction massage. The final study found a nonsignificant advantage of spinal manipulation therapy over myofascial therapy for pain and disability reduction. All three studies had low- to moderate-quality scores $(13 \%, 25 \%$, and $63 \%)$.

Brosseau et $\mathrm{al}^{20}$ identified eleven trials regarding massage for the treatment of acute, subacute, or chronic nonspecific low back pain. However, the authors reported on only four trials, comprising 954 participants, which scored 3 or more on the Jadad scale ${ }^{21}$ (high methodological quality). They found statistically significant results in favour of pain reduction in comparison to sham laser treatment but only "clinically important" ${ }^{20}$ results in decreased disability and symptoms for massage (structural, therapeutic, and relaxation massage) in comparison to usual care and sham laser treatment.

Lewis and Johnson ${ }^{10}$ identified seven relevant studies, with a total of 787 participants. These studies were critically appraised using the Centre for Reviews and Dissemination methodological scale. ${ }^{22}$ The included studies scored between 3 and 7 out of a maximum score of 9 . The Centre for Reviews and Dissemination methodological scale was modified to a maximum score of 9 (rather than the original maximum score of 11) by Lewis and Johnson, as they recognized the inability to blind practitioners and clients for this type of intervention (loss of 2 points). ${ }^{10}$ There was considerable variation between the studies, limiting the ability to compare the findings.
Therapeutic massage resulted in better pain and disability scores by end of trial than sham laser, and was found to be superior to self-care, acupuncture, exercise and education, and muscle relaxation. Soft tissue manipulation (six sessions, over a 1-month period) was more effective in terms of reducing disability and pain than exercise with posture education or treatment with sham laser for people with subacute low back pain. Massage (three 30-minute sessions per week for 3 weeks) was better than mental relaxation, while massage (two 30-minute sessions per week for 5 weeks) reduced pain in comparison with standard medical care (pharmacology and chronic pain education). The authors concluded that, as therapeutic massage was superior to comparison groups in only three of seven studies, the effectiveness of massage to relieve low back pain was inconclusive.

Lin et $\mathrm{al}^{23}$ identified one RCT with low risk of bias, comprising 579 participants, which investigated the costeffectiveness of massage therapy as compared to general practice (GP) health care. This study indicated that massage alone was less effective and more expensive from the health care sector's perspective than GP care. However, when exercise and behavioral counseling were added to massage, it was more cost-effective than GP care.

Therefore, to summarize this research evidence, massage therapy may offer some positive benefits in terms of reduction in pain and disability in the short term, when compared to sham and placebo interventions. However, this was not the case when massage therapy was compared with spinal manipulative therapy. There is equivocal evidence of effectiveness of massage therapy when compared to acupuncture, exercise and education, and relaxation. There is limited evidence on the cost-effectiveness of massage therapy when compared to other standard interventions for nonspecific low back pain.

\section{Discussion}

The aim of this umbrella review was to provide a synthesis and judgment of best available research evidence related to the effectiveness of massage therapy for the treatment of nonspecific low back pain. With increasing consumption of CAM therapies, in an era of evidence-based practice, it is only appropriate to investigate the evidence underpinning the effectiveness of CAM therapies.

The systematic reviews included in this umbrella review ranged from poor methodological quality ${ }^{10,16}$ to moderate, ${ }^{18,19}$ good, ${ }^{4,15,20}$ or excellent methodological quality. ${ }^{17,23}$ Therefore, we recommend that caution be used when interpreting the conclusions of these reviews, as the primary research relat- 
ing to massage and non-specific low back pain, for the most part, had a high risk of bias.

While there are a number of systematic reviews investigating the effectiveness of massage therapy for nonspecific low back pain, there is mixed and conflicting evidence on outcomes from massage therapy. There is emerging evidence that massage may be an effective treatment option for treating low back pain when compared to placebo or sham therapies and other interventions (such as relaxation techniques) in improving short-term pain and disability. The role of massage as a moderately effective nonpharmacological treatment option has also been discussed by Chou et $\mathrm{al}^{24}$ and Imamura et $\mathrm{al}^{8}$ as a recommendation for chronic low back pain therapy.

The evidence is contradictory for the effectiveness of massage when compared to other popular treatment options, such as standard medical care, mobilization, and acupuncture in improving short-term pain and disability. Spinal manipulative therapy seems consistently to provide better outcomes when compared to massage therapy. There was no evidence found for the long-term (beyond 6 months) effectiveness of massage therapy.

The methodology underpinning the primary research, which informed the systematic reviews, was, for the most part, classified as weak. This is a significant issue that has plagued the evidence base for massage therapy and has also been acknowledged by other researchers (Airaksinen et $\mathrm{al}^{25}$ ). The methodological issues reported by the systematic reviews include small sample size, lack of adequate blinding of assessors, and varied intervention parameters and outcome measures. This is demonstrated by the finding that only one of the nine included systematic reviews was able to undertake a meta-analysis of the included primary literature. ${ }^{18}$ This was due to the variability in the description of intervention parameters, operational definition of massage therapy, comparators, and outcome measures utilized in the remaining eight included systematic reviews.

The poor quality of the primary research evidence base may be partly due to the conflict between what occurs in clinical practice and rigorous standards required within research settings. In a clinical practice context, massage therapy may often be offered as a "package of care" in addition to advice and education and using a combination of modalities. However, in a research context, a package of care is rarely offered in order to avoid cointervention bias. Therefore, the primary research undertaken may not truly capture and replicate what occurs in a clinical practice context. This is a challenge and a limitation when undertaking and interpreting findings of research evidence for massage therapy.

\section{Limitations of this review}

This umbrella review, like any other research, has its limitations, and these need to be acknowledged in the context of the findings. Firstly, while all attempts were made to interrogate and access all relevant literature, it is possible some publications may have been missed in the search process. This is especially relevant for CAM topics, as publications in other languages, originating from countries where English may not be a first language, such as the People's Republic of China and India, may not be captured in Western databases. Secondly, as there was a lack of clarity around the type, use, and comparators of massage therapy in these systematic reviews, the heterogeneity made it impossible to combine the findings across all included systematic reviews and come to an absolute conclusion. Thirdly, one of the recurrent issues when interpreting these systematic review findings was the imperfect primary research designs included in these reviews. Several of the primary research studies had poor evaluations with several methodological issues (such as lack of adequate descriptions of interventions and poor long-term follow-up).

\section{Conclusion}

\section{Implications for practice}

The findings of this umbrella review indicate that massage may be an effective treatment option in the short term when compared to placebo and some active treatment options (such as relaxation). However, there are conflicting and contradictory findings for the effectiveness of massage therapy for the treatment of nonspecific low back pain when compared against other manual therapies (such as mobilization, standard medical care, and acupuncture). Given that there were no reported side effects or adverse events as a result of massage therapy, it may be considered as a viable treatment option, provided that cost implications are considered.

The diversity and complexity of the evidence base for the effectiveness of massage therapy for the treatment of nonspecific low back pain underscores the importance of a collaborative, patient-centered decision making process between the patient and the health professional, which is informed by best available evidence. In addition to this, sound clinical reasoning, expertise of individual health professionals, and health outcome data, collected using rigorous outcome measures, should underpin the integration of the findings from this umbrella review into clinical practice. 
These processes build on the philosophy of evidence-based practice in health care.

\section{Implications for research}

Massage therapy seems to be a well-researched field of therapy within CAMs. However, there are key knowledge gaps in the literature that need to be addressed. Further research is required to unpack the "black box" of massage therapy, as there is ambiguity on the operational definition of massage therapy. Various systematic reviews defined and searched for many different types and modalities of massage, with some discussing massage techniques as a separate modality and some considering massage therapy as part of a suite of interventions. This variability extended to massage therapy parameters such as dosage, duration, and intervention protocols. There is a scarcity of data on the cost-effectiveness of massage therapy for the treatment of nonspecific low back pain. As there is currently a dearth of high-quality/low risk of bias primary research on the effectiveness of massage for the treatment of nonspecific low back pain, further research, such as RCTs, with sound methodological rigor, are required. While there are a number of systematic reviews investigating the effectiveness of massage therapy for nonspecific low back pain, the mechanism underlying its action remains elusive. While physiological and psychotherapeutic models have been proposed, the precise mechanism of action continues to be debated, requiring ongoing further research.

\section{Acknowledgment}

The authors gratefully acknowledge Ms Khushnum Pastakia for her assistance and feedback during the preparation of this manuscript.

\section{Disclosure}

The funding for the conduct of this umbrella review was provided by the Australian Association of Massage Therapists. The authors report no other conflicts of interest in this work.

\section{References}

1. Kohn LT, Corrigan JM, Donaldson MS. To err is human: building a safer health system. Institute of Medicine; 2000. Available from: http://wps. pearsoneducation.nl/wps/media/objects/13902/14236351/H\%2007_ To\%20Err\%20Is\%20Human.pdf. Accessed September 14, 2012.

2. Sackett DL, Rosenberg WM, Gray JA, Haynes RB, Richardson WS. Evidence based medicine: what it is and what it isn't. BMJ. 1996;312: $71-72$.
3. Furlan AD, Brosseau L, Imamura M, Irvin E. Massage for low-back pain: a systematic review within the framework of the Cochrane Collaboration Back Review Group. Spine (Phila PA 1976). 2002;27:1896-1910.

4. Furlan AD, Yazdi F, Tsertsvadze A, et al. A systematic review and meta-analysis of efficacy, cost-effectiveness, and safety of selected complementary and alternative medicine for neck and low-back pain. Evid Based Complement Alternat Med. 2012;2012:953139.

5. Furlan A, Imamura M, Dryden T, Irvin E. Massage for low back pain: an updated systematic review within the framework of the Cochrane Back Review Group. Spine (Phila Pa 1976). 2009;34(16):1669-1684.

6. Andersson GB. Epidemiological features of chronic low-back pain. Lancet. 1999;354:581-585.

7. Hestbaek L, Leboeuf-Yde C, Manniche C. Low back pain: what is the long-term course? A review of studies of general populations. Eur Spine J. 2003;12:149-165.

8. Imamura M, Furlan AD, Dryden T, Irvin E. Evidence-informed management of chronic low back pain with massage. Spine $J$. 2008;8(1): 121-133.

9. MacLennan AH, Myers SP, Taylor AW. The continuing use of complementary and alternative medicine in South Australia: costs and beliefs in 2004. Med J Aust. 2006;184:27-31.

10. Lewis M, Johnson MI. The clinical effectiveness of therapeutic massage for musculoskeletal pain: a systematic review. Physiotherapy. 2006;92:146-158.

11. Merlin T, Weston A, Tooher R. Extending an evidence hierarchy to include topics other than treatment: revising the Australian 'levels of evidence'. BMC Med Res Methodol. 2009;9(34).

12. Moyer CA, Rounds J, Hannum JW. A meta-analysis of massage therapy research. Psychol Bull. 2004;130:3-18.

13. Liberati A, Altman DG, Tetzlaff J, et al. The PRISMA statement for reporting systematic reviews and meta-analyses of studies that evaluate healthcare interventions: explanation and elaboration. $B M J$. 2009;339:b2700.

14. Centre for Evidence Based Medicine. Systematic Review Critical Appraisal Sheet. Oxford: University of Oxford; 2005. Available from: http://www.cebm.net/index.aspx?o=1157. Accessed November 16, 2012.

15. Pengel HM, Maher CG, Refshauge KM. Systematic review of conservative interventions for subacute low back pain. Clin Rehabil. 2002;16: 811-820.

16. Bronfort G, Haas M, Evans RL, Bouter LM. Efficacy of spinal manipulation and mobilization for low back pain and neck pain: a systematic review and best evidence synthesis. Spine J. 2004;4:335-356.

17. Ernst E, Posadzki P, Lee MS. Reflexology: an update of a systematic review of randomised clinical trials. Maturitas.2011;68:116-120.

18. Kim YC, Lee MS, Park ES, Lew JH, Lee BJ. Acupressure for the treatment of musculoskeletal pain conditions: a systematic review. J Musculoskelet Pain. 2012;20:116-121.

19. van Middelkoop M, Rubinstein SM, Kuijpers T, et al. A systematic review on the effectiveness of physical and rehabilitation interventions for chronic non-specific low back pain. Eur Spine J. 2011;20:19-39.

20. Brosseau L, Wells GA, Poitras S, et al. Ottawa Panel evidence-based clinical practice guidelines on therapeutic massage for low back pain. J Bodyw Mov Ther. 2012;16:424-455.

21. Jadad AR, Moore RA, Carroll D, Jenkinson C, Reynolds JM, Gavaghan DJ, McQuay HJ. Assessing the quality of reports of randomized clinical trials: is blinding necessary? Control Clin Trials. 1996;17:1-12.

22. Khan KS, Ter Riet G, Glanville J, Sowden AJ, Kleijnen J, editors. The NHS Centre for Reviews and Dissemination (CRD). Undertaking systematic reviews of research on effectiveness. CRD's guidance for carrying out or commissioning reviews. 2nd ed. CRD Report No. 4. York: NHS Centre for Reviews and Dissemination, University of York; 2000.

23. Lin CW, Haas M, Maher CG, Machado LA, van Tulder MW. Costeffectiveness of guideline-endorsed treatments for low back pain: a systematic review. Eur Spine J. 2011;20:1024-1038. 
24. Chou R, Qaseem A, Snow V, et al; Clinical Efficacy Assessment Subcommittee of the American College of Physicians; American College of Physicians; American Pain Society Low Back Pain Guidelines Panel. Diagnosis and treatment of low back pain: a joint clinical practice guideline from the American College of Physicians and the American Pain Society. Ann Intern Med. 2007;147(7):478-491.
25. Airaksinen O, Brox JI, Cedraschi C, et al; COST B13 Working Group on Guidelines for Chronic Low Back Pain. Chapter 4. European guidelines for the management of chronic nonspecific low back pain. Eur Spine J. 2006;15 Suppl 2:S192-S300.

\section{Publish your work in this journal}

The International Journal of General Medicine is an international, peer-reviewed open-access journal that focuses on general and internal medicine, pathogenesis, epidemiology, diagnosis, monitoring and treatment protocols. The journal is characterized by the rapid reporting of reviews, original research and clinical studies across all disease areas.
A key focus is the elucidation of disease processes and management protocols resulting in improved outcomes for the patient.The manuscript management system is completely online and includes a very quick and fair peer-review system. Visit http://www.dovepress.com/ testimonials.php to read real quotes from published authors.

Submit your manuscript here: http://www.dovepress.com/international-journal-of-general-medicine-journal 